\title{
Self reported respiratory symptoms and diseases among hairdressers
}

Timo Leino, Lauri Tammilehto, Ritva Luukkonen, Henrik Nordman

\begin{abstract}
Objectives-Hairdressers are exposed to many irritative and allergenic substances capable of causing occupational respiratory symptoms and diseases. The self reported prevalence of respiratory symptoms and diseases was studied, and the risks among hairdressers compared with saleswomen was estimated.

Methods-A cross sectional prevalence study of respiratory symptoms and diseases was carried out among hairdressers

Conclusions-Our results indicate an increased prevalence of upper and lower respiratory symptoms among hairdressers. Allergenic and irritative chemicals in hairdressing are likely candidates explaining the difference found between the hairdressers and controls. Work related reasons should be considered when a hairdresser presents with airway symptoms. Preventive actions are needed to improve the working conditions and personal protection.
\end{abstract} and supermarket saleswomen, with a computer assisted telephone interview method (CATI). The study population comprised all the female hairdressers and supermarket saleswomen aged 15-54 years in the Helsinki metropolitan area, Finland. Disproportionate random samples of female hairdressers and saleswomen were drawn from the trade union membership registers. The interviews were carried out between February and April 1994. A response rate of $80.5 \%$ (355/440) was obtained for hairdressers and $82.2 \%(583 / 709)$ for saleswomen. Atopy, smoking, chronic illnesses, type of work, working hours, working conditions, personal and professional use of hair products, and the use of personal protective devices were assessed. The outcome variables were self reported symptoms of the upper and lower respiratory tract. These were used to define chronic bronchitis, and asthma, laryngitis, and allergic rhinitis diagnosed by a physician.

Results-There was a considerable difference in the prevalence of chronic bronchitis; $6.8 \%$ in hairdressers versus $1.9 \%$ in saleswomen. The odds ratio (OR) adjusted for age, smoking, and atopy for chronic bronchitis indicated an increased risk of chronic bronchitis (OR 4.8, 95\% confidence interval (95\% CI) 2.2 to 10.1). No association was found between work as a hairdresser and asthma, laryngitis, and allergic rhinitis. Also the prevalence of rhinitis, rhinitis with eye symptoms, cough with phlegm, dyspnoea, and dyspnoea accompanied by cough was increased among hairdressers. The corresponding adjusted risk ORs were 1.7 (95\% CI 1.3 to 2.3) for rhinitis, 1.9 (95\% CI 1.4 to 2.6 ) for rhinitis with eye symptoms, 1.4 (CI 1.1 to 1.9) for cough with phlegm, $1.5(95 \% \mathrm{CI} 1.0$ to 2.2$)$ for dyspnoea, and $1.6(95 \%$ CI 1.0 to 2.7$)$ for dyspnoea with cough.

\section{(Occup Environ Med 1997;54:452-455)}

Keywords: asthma; chronic bronchitis; occupational

The prevalence of asthma and other respiratory illnesses and symptoms in populations varies widely according to environmental factors, with occupational exposures being among the most important. ${ }^{1}$ Hairdressers are exposed to a variety of irritative and allergenic substances, ${ }^{2}$ which can cause airway symptoms and diseases related to work. ${ }^{34}$ Hair lacquers and permanent wave solutions can irritate airways and worsen the symptoms of people with reactive airways or asthma. ${ }^{5-11}$ Several case reports have been published on occupational asthma caused by persulphate salts in hair bleaches. ${ }^{12-16}$ Henna can also cause asthma. ${ }^{13-17}$ Polyvinylpyrrolidone in hair lacquers has been suspected of causing alveolitis ${ }^{18} 19$ and lung granulomatosis (thesaurosis), ${ }^{20}$ but the results have been controversial and upon closer scrutiny the association is weak or lacking. ${ }^{22} 23$

Our aim was to study the self reported prevalence of respiratory symptoms and diseases, and to estimate their risk among hairdressers compared with saleswomen.

Subjects and methods

As $96 \%$ of the 13200 hairdressers in Finland were women according to the 1990 census, only women were selected for this study. The study population comprised all the female hairdressers and saleswomen aged 15-54 years, presently living and working in the Helsinki metropolitan area (City of Helsinki, Espoo, Vantaa and Kauniainen, female population 464000 in 1994). A stratified disproportionate random sample in five-year age groups ket saleswomen was drawn from the trade union membership registers. The selection of the control group was based on a similar socioeconomic class as that of the hairdressers. of 500 female hairdressers and 1000 supermar- 
The controls were required not to work on tasks in which the exposure to chemicals or organic materials is known to be substantial. Those controls who had been working previously as hairdressers or did hairdressing as a part time job or at home were also excluded. After removing those who had not been working in their profession within the three months before the study started, the final samples consisted of 440 hairdressers and 915 saleswomen. All participants received a letter stating the purpose of the study before they were contacted by phone. Participation was voluntary. The study protocol was approved by the ethics committee of the Finnish Insitute of Occupational Health.

The study was conducted by a computer assisted telephone interview system (CATI) from February to April 1994. We used the CASES software package for programming the interview protocol. ${ }^{24}$ The program was tested in the pilot study and corrected accordingly. All the interviewers received one day of training before the actual study started. Only qualified and experienced interviewers were used. The quality control was performed by monitoring part of the interviews. The interview always followed the preprogrammed question pattern and the answers were simultaneously recorded for further statistical analysis. Open answers were coded by the researchers. The interviews were performed at the most suitable times for the interviewees to guarantee their full participation. ${ }^{25}$

The interviewees were asked about their personal characteristics including atopy and smoking habits, perceived chronic illnesses, medication, previous occupations, type of work, working hours a week, working conditions, personal and professional use of hair products, and use of personal protective devices.

Respiratory diseases were probed with the following questions: Do you have chronic respiratory disease, asthma, allergic rhinitis, laryngitis, or vocal chord changes (here grouped together) and was it diagnosed by a physician? Questions on respiratory symptoms included: Do you have cough and phlegm? Do you have shortness of breath (dyspnoea)? Is your dyspnoea accompanied by wheezing, cough, phlegm, hoarseness, or other vocal problems (questions again grouped together)? Do you have rhinitis - that is, sneezing, a stuffy nose or a runny nose, which has not been caused by the common cold? Is your rhinitis accompanied by eye symptoms? Do you have hoarseness or other vocal problems? Each question asked the duration of the symptoms and their relation to environmental and occupational factors.

Outcome variables were self reported symptoms of the upper and lower respiratory tract and respiratory diseases (asthma, laryngitis, and allergic rhinitis) diagnosed by a physician. The identification of the cases of chronic bronchitis was based on symptoms. A subject had to have cough and phlegm for at least three months of the year, for more than two years, and should not have had asthma diagnosed. ${ }^{26}$
Table 1 Personal characteristics of the study groups

\begin{tabular}{lll}
\hline & $\begin{array}{c}\text { Hairdressers } \\
(n=335) \%\end{array}$ & $\begin{array}{l}\text { Saleswomen } \\
(n=583) \%\end{array}$ \\
\hline Age (y): & & \\
$16-24$ & 25.3 & 15.6 \\
$25-34$ & 42.8 & 24.5 \\
$35-44$ & 17.5 & 32.7 \\
$45-55$ & 14.4 & 27.1 \\
Atopy: & & \\
Yes & 43.4 & 44.8 \\
No & 56.6 & 55.2 \\
Smoking: & & \\
Non-smoker & 41.7 & 34.7 \\
Ex-smoker & 11.0 & 16.1 \\
Current smoker & 47.3 & 49.2 \\
\hline
\end{tabular}

* Based on separate questions about: Have you ever had-asthma, allergic rhinitis, or atopic dermatitis?

Atopy was considered to be present if the subject reported that she had had asthma, allergic rhinitis, or atopic dermatitis at any time in her life.

A subject was defined as a non-smoker if she had never smoked daily for as long as one year. Smokers were defined as subjects currently smoking at least one cigarette, cigar, or pipe each day. In the final analysis, ex-smokers were grouped together with current smokers.

\section{STATISTICAL METHODS}

The multivariate logistic regression analyses were used for both respiratory symptoms and diseases to give the odds ratios (ORs) adjusted for age, smoking, and atopy, and the $95 \%$ confidence intervals (95\% CIs). All calculations were performed with the SAS statistical package. ${ }^{26}$

\section{Results}

Of the study group 60 did not meet the selection criteria, whereas in the control group 85 people were not eligible for the study. The telephone numbers of 60 hairdressers and 204 saleswomen could not be traced. Of the hairdressers, 10 refused to participate in the interview and 15 could not be contacted. The corresponding figures for the saleswomen were 63 and 65 . A response rate of $80.5 \%$ (355/440) was obtained for the hairdressers and $82.2 \%$ (583/709) for the saleswomen.

The hairdressers were younger than the controls. The mean (SD) age of the hairdressers was 31.5 (9.3) years compared with $36(9.8)$ years in the control group. The hairdressers and saleswomen were selected from the same geographical area, and they were all city dwellers. About half of the subjects in both groups were current smokers. Never-smokers were overrepresented in the hairdressers' group, whereas the proportion of ex-smokers was higher in the control group. The lifetime occurrence of atopic diseases (asthma, allergic rhinits, and atopic dermatitis) did not differ notably between the study group and controls (table 1).

The prevalences of rhinitis, rhinitis with eye symptoms, cough with phlegm, dyspnoea, and dyspnoea with cough, were significantly higher among the hairdressers than among the controls. The OR for these symptoms varied between 1.5 and 1.9 among hairdressers even 
Table 2 Prevalences and risk ORs for respiratory symptoms and diseases among hairdressers compared with saleswomen

\begin{tabular}{|c|c|c|c|c|}
\hline & $\begin{array}{l}\text { Hairdressers } \\
(n=355) \%\end{array}$ & $\begin{array}{l}\text { Saleswomen } \\
(n=583) \%\end{array}$ & $\begin{array}{l}\text { OR* } \\
(95 \% C I)\end{array}$ & $\begin{array}{l}\text { ORt } \\
(95 \% C I)\end{array}$ \\
\hline \multicolumn{5}{|l|}{ Symptoms: $\ddagger$} \\
\hline Dyspnoea: & 16.1 & 13.2 & $1.3(0.9$ to 1.8$)$ & 1.5 (1.0 to 2.2$)$ \\
\hline wheezing & 4.8 & 5.8 & $0.9(0.5$ to 1.6$)$ & $1.0(0.5$ to 1.8$)$ \\
\hline Dyspnoea and cough & 10.1 & 7.5 & $1.4(0.9$ to 2.2$)$ & $1.6(1.0$ to 2.7$)$ \\
\hline Dyspnoea and phlegm & 7.9 & 6.2 & $1.3(0.8$ to 2.2$)$ & $1.6(0.9$ to 2.7$)$ \\
\hline \multicolumn{5}{|l|}{ Dyspnoea and } \\
\hline hoarseness & 9.6 & 7.9 & $1.2(0.8$ to 2.0$)$ & $1.4(0.9$ to 2.3$)$ \\
\hline Cough and phlegm & 35.8 & 30.4 & $1.3(1.0$ to 1.7$)$ & $1.4(1.1$ to 1.9$)$ \\
\hline Hoarseness & 29.6 & 30.9 & $0.9(0.7$ to 1.3$)$ & $0.9(0.7$ to 1.3$)$ \\
\hline Rhinitis & 62.3 & 50.6 & $1.6(1.2$ to 2.1$)$ & $1.7(1.3$ to 2.3$)$ \\
\hline \multicolumn{5}{|l|}{ Rhinitis and eye } \\
\hline symptoms & 32.7 & 22.8 & $1.6(1.2$ to 2.2$)$ & $1.9(1.4$ to 2.6$)$ \\
\hline \multicolumn{5}{|l|}{ Diseases: $\$$} \\
\hline Asthma & 4.2 & 5.7 & $0.7(0.4$ to 1.4$)$ & $0.9(0.5$ to 1.7$)$ \\
\hline Chronic bronchitis $\ddagger$ & 6.8 & 1.9 & $3.8(1.8$ to 7.8$)$ & $4.8(2.2$ to 10.1$)$ \\
\hline Laryngitis & 5.1 & 5.7 & $0.9(0.5$ to 1.6$)$ & $0.8(0.5$ to 1.6$)$ \\
\hline Allergic rhinitis & 16.9 & 19.9 & $0.8(0.6$ to 1.2$)$ & $0.8(0.5$ to 1.1$)$ \\
\hline
\end{tabular}

^ Not adjusted.

† Adjusted for age, smoking, and atopy.

¥ Based on a yes answer to certain symptom questions.

$\oint$ Based on a yes answer to the question: have you a given condition diagnosed by a physician?

Table 3 Risk ORs of having a respiratory disease by determinants yeilded by the logistic regression model

\begin{tabular}{lll}
\hline Disease & Determinant & OR (95\% CI) \\
\hline Asthma & Hairdressing & $0.9(0.5$ to 1.7$)$ \\
& Smoking & $0.9(0.5$ to 1.6$)$ \\
& Age & $1.0(1.0$ to 1.1$)$ \\
Atopy & $6.5(3.1$ to 13.7$)$ \\
& Hairdressing & $5.5(2.5$ to 12.0$)$ \\
& Smoking: & \\
& Never smoker & 1.0 \\
& Ex-smoker & $0.6(0.1$ to 2.8$)$ \\
& $1-19$ Cigarettes/day & $1.7(0.7$ to 4.3$)$ \\
& Ag0 Cigarettes/day & $6.2(2.4$ to 15.8$)$ \\
Laryngitis & Atopy & $1.0(1.0$ to 1.1$)$ \\
& Hairdressing & $2.1(1.0$ to 4.3$)$ \\
& Smoking & $0.8(0.5$ to 1.6$)$ \\
& Age & $1.1(0.6$ to 2.0$)$ \\
Allergic rhinitis & Atopy & $1.0(0.9$ to 1.0$)$ \\
& Hairdressing & $2.2(1.2$ to 3.9$)$ \\
& Smoking & $0.8(0.5$ to 1.1$)$ \\
& Age & $0.8(0.6$ to 1.3$)$ \\
& Atopy & $1.0(0.9$ to 1.0$)$ \\
& & $3.2(2.2$ to 4.5$)$ \\
\hline
\end{tabular}

after adjustment for age, atopy, and smoking in the logistic regression model (table 2).

There was a considerable difference in the prevalence of chronic bronchitis, whereas the prevalences for other respiratory diseases (asthma, laryngitis, and allergic rhinitis) were similar. The OR adjusted for age, smoking, and atopy for chronic bronchitis did indicate an increased risk of chronic bronchitis (OR 4.8, 95\% CI 2.2 to 10.1 , table 2 ).

Table 3 shows the multinomial analysis of the determinants of the outcomes in the logistic regression model. Atopy was a strong independent determinant in all of the studied respiratory diseases diagnosed by a physician. The OR for asthma was 6.5 , for chronic bronchitis and laryngitis 2.2 , and for allergic rhinitis 3.2. Smoking increased the risk of chronic bronchitis, as expected, and the risk was highest in the heavy $(20+)$ smokers subgroup $(n=$ 93) of hairdressers (OR $6.2,95 \%$ CI 2.4 to 15.8). The work as a hairdresser was a significant determinant only for chronic bronchitis.

\section{Discussion}

Hairdressers are exposed to a broad range of irritative and allergenic chemicals in their work. Suprisingly few studies on the prevalence of respiratory symptoms and diseases, however, have been published. We used the computerassisted telephone interview method (CATI), which had a significant advantage over postal surveys. It allowed detailed questions on work variables, perceived respiratory symptoms, and conditions diagnosed by a physician. A postal questionnaire would have been cumbersome to fill out and would possibly have unfavourably affected compliance as well as the quality of the collected data. Hairdressers recalling airway symptoms better than the saleswomen is a conceivable source of bias. We tried to avoid this by structuring the interview; we asked whether the symptoms could be related to work only after inquiring about other possible nonoccupational reasons for the symptoms.

We have shown that hairdressers have a higher risk than saleswomen of chronic bronchitis, asthma-like symptoms, and rhinitis accompanied with irritative eye symptoms. Experimental studies of the harmful effects of hair care chemicals on the airway epithelium provide a likely explanation of the increased prevalence of respiratory symptoms among hairdressers. ${ }^{5-11}$ Although the OR for chronic bronchitis (4.8) was high, the OR for asthma was not increased. When a hairdresser develops asthma she often has to leave her work. The increased prevalence of asthma like symptoms among hairdressers, however, presents the possibility of an increased risk of asthma-a subject for further investigation. Questionnaire studies have their limitations in detecting asthma and chronic bronchitis, as do physicians when diagnosing these conditions. ${ }^{28} 29$

In both study groups the prevalence of asthma is close to the estimated prevalence of asthma in the general population in the Nordic countries. ${ }^{30}$ The prevalence of chronic bronchitis among hairdressers is higher than among all women in the Nordic countries, and very similiar to that which has been reported among non-smoking agricultural women $(7.7 \%)$ in Finland. ${ }^{30}{ }^{31}$ Our results are consistent with the increased prevalence of respiratory symptoms among hairdressers found in the study of Palmer $e t a l{ }^{3}$ who reported abnormal respiratory symptoms consistent with a chronic obstructive lung disorder in $12 \%$, and physician diagnosed chronic respiratory disease in $8 \%$ of hairdressers compared with $7.6 \%$ and $12 \%$, respectively, among the controls. The difference in respiratory disorders between the study cohort and controls became significant only after the mild cases were included in the analysis.

In conclusion, the study provides some further insights into the pattern of respiratory symptoms among hairdressers. Hairdressers are at increased risk of symptoms of both the upper and lower respiratory tract. Although it is not possible to draw any definite conclusions about the causal relations, it is likely that the increased prevalence of respiratory symptoms results from the occupational exposure to hairdressing chemicals. The prevalence of respiratory diseases and symptoms are unacceptably high. Future studies should focus on the 
prevention of those respiratory symptoms related to work. Detailed studies on working conditions and exposures in hairdressing salons are needed.

This study was supported by a grant from the Finnish Work Environment Fund. We thank Dr Helena Piirainen, Mr Asko Lötionen, the staff of the CATI Unit at the Kuopio Regional Institute of Occupational Health for their excellent help with data collection and processing, and Ms Terttu Kaustia for the language revision.

1 Meredith S, Nordman H. Occupational asthma: measures of frequency from four countries. Thorax 1995;51:435.

2 Wenninger JA, McEwen GN Jr, eds. International cosmetic ingredient dictionary. Washington, DC: The Cosmetic, Toiletry, and Fragrances Association, 1993:937-97.

3 Palmer A, Renzetti A, Gillam D. Respiratory disease prevalence in cosmetologists and its relationship to aeroso sprays. Environ Res 1979;19:136-53.

4 Blainey A, Ollier S, Cundell D, Smith R, Davies R. Occupational asthma in hairdressing salon. Thorax 1986;41:42-50.

5 Swift D, Zuskin E, Bouhuys A. Respiratory deposition of hair spray aerosol and acute lung function changes. $\mathrm{Am}$ Rev Respir Dis 1976;113(suppl):96.

6 Friedman M, Dougherty R, Nelson SR, White RP, Sackner MA, Wanner A. Acute effects of aerosol hair spray on tracheal mucociliary transport. Am Rev Respir Dis 1977;116: cheal mu.

7 Schlueter DP, Soto RJ, Baretta ED, Herrmann AA Ostrander LE, Stewart RD. Airway response to hair spray in normal subjects and subjects with hyperreactive airways Chest 1979;75:544-8.

8 Zuskin E, Bouhuys A, Beck G. Hair sprays and lung function. Lancet 1978;ii: 1203

9 Zuskin E, Loke J, Bouhuys A. Helium-oxygen flow-volume curves in detecting acute response to hair sprays. Int Arch Occup Environ Health 1981;49:41-4.

10 Borum P, Loekkegaard N, Holten A. Nasal mucociliary clearance in hairdressers. Clinical Otolaryngology and Allied Sciences 1984;9:329-34.

11 Schwartz HJ, Arnold JL, Strohl KP. Occupational allergic rhinitis in the hair care industry: reactions to permanent rhinitis in the hair care industry: reactions

12 Gaultier R, Gervais P, Mellerio F. Two cases of occupational asthma and urticaria in hairdressers: persulphate and silk. asthma and urticaria in hairdressers: persulphate

13 Pepys J, Hutchcroft BJ, Breslin ABX. Asthma due to inhaled chemical agents - persulphate salts and henna in hairdressers. Clin Allergy 1976;6:399-404.
14 Pankow W, Hein H, Bittner K, Wichert P. Persulfate asthma in hairdressers. Pneumologie 1989;3:173-5.

15 Schwaiblmair M, Baur X, Fruhmann G. Bronchial asthma caused by hairbleach in a hairdresser. Dtsch Med Wochenschr 1990;18:695-7.

16 Parra FM, Igea JM, Quirce S, Ferrando MC, Martin JA, Losada E. Occupational asthma in a hairdresser caused by persulphate salts. Allergy 1992;47:656-60.

17 Starr J, Yunginger J, Brahser G. Immediate type I asthmatic response to henna following occupational exposure in hairdressers. Ann Allergy 1982;48:98-9.

18 Stringer G, Hunter S, Bonnabeau R. Hypersensitivity pneumonitis following prolonged inhalation of hair spray. $A m$ Med Assoc 7 1977;238:888-9.

19 Gebbers J-O, Tetzner C, Burkhardt A. Alveolitis due to hair-spray. Virchows Archiv A Pathological Anatomy and Histopathology 1979;382:323-38.

20 Bergmann $\mathrm{M}$, Flance IJ, Blumenthal $\mathrm{H}$. Thesaurosis following inhalation of hairspray: a clinical and experimental study. $N$ Engl f Med 1958;258:471.

21 Gelfand $\mathrm{H}$. Respiratory allergy due to chemical compounds encountered in the rubber, lacquer, shellac and beauty culture industries. $\mathcal{F}$ Allergy 1963;34:374-81.

22 Renzetti A, Conrad J, Watanabe S, Palmer A, Armstrong J Thesaurosis - from hairspray exposure - a nondisease. Environ Res 1980;22:130-8.

23 Ameille J, Pages MG, Capron F, Proteau J, Rochemaure J. Pathologie respiratoire induite par l'inhalation de laque capillaire. Rev Pneumol Clin 1985;41:325-30.

24 CASES. Computer-assisted survey methods program: user's guide to CASES. Berkeley, CA: University of California 1989.

25 Frey JH. Survey research by phone, 2nd ed.New York: Sage Publication, 1989. (Sage Library of Social Research 150.)

26 Medical Research Council's Committee on the Aetiology of Chronic Bronchitis. Standardized questionnaires on respiratory symptoms. BMF 1960;ii: 1665 .

27 SAS / STAT. User's guide, version 6, 4th ed. Vol 2. Cary, NC. SAS Institute, 1990

28 Torén K, Brisman J, Järvholm B. Asthma and asthma-like symptoms in adults assessed by questionnaires - a literature review. Chest 1993;104:600-18.

$29 \mathrm{Li}$ JTC, O'Connell EJO. Clinical evaluation of asthma. Ann Allergy Asthma Immunol 1996;76:1-13.

30 Bakke P, Blöndal T, Haahtela T, Lundgren R, Rasmussen FV, Walstad R. Care of obstructive lung diseases in the Nordic countries. Eur Respir Rev 1995;5:350-5.

31 Terho EO, Husman K, Vohlonen I. Prevalence and incidence of chronic bronchitis and farmer's lung with respect to age, sex, atopy and smoking. Eur $\mathcal{F}$ Respir Dis $1987 ; 71: 19-28$

\section{Occupational and Environmental Medicine and the electronic age}

OEM has an Email address which is 100632.3615@compuserve.com. We welcome contact by Email, including letters to the editor. Some of our reviewers already send us their reports by Email, helping to speed up the peer review process.

We are moving towards electronic publishing and for some months now we have been asking authors to send us their revised papers on disk as well as a hard copy. I am delighted to report that nearly all our authors are managing to comply with this request. Oddly enough, the few authors who have not sent us a disk version of their revised papers have been almost exclusively from the United Kingdom. I would be interested in suggestions for why this might be. Perhaps United Kingdom based authors read our correspondence and instructions less assiduously? Watch for revised Instructions to Authors.

The Editor 